\title{
MUC16 affects the biological functions of ovarian cancer cells and induces an antitumor immune response by activating dendritic cells
}

\author{
Yan Zhai^, Qi Lu, Tong Lou, Guangming Cao, Shuzhen Wang, Zhenyu Zhang \\ Department of Obstetrics and Gynecology, Beijing Chao-Yang Hospital, Capital Medical University, Beijing, China \\ Contributions: (I) Conception and design: Y Zhai, Z Zhang, S Wang; (II) Administrative support: S Wang; (III) Provision of study materials or \\ patients: Q Lu, T Lou; (IV) Collection and assembly of data: G Cao; (V) Data analysis and interpretation: Y Zhai, G Cao; (VI) Manuscript writing: \\ All authors; (VII) Final approval of manuscript: All authors. \\ Correspondence to: Zhenyu Zhang; Shuzhen Wang. Department of Obstetrics and Gynecology, Beijing Chao-Yang Hospital, Capital Medical \\ University, No. 8 Gongti South Road, Chaoyang District, Beijing 100020, China. Email: zhenyuzhang2000@163.com; darrywang2003@163.com.
}

\begin{abstract}
Background: Ovarian cancer is the $5^{\text {th }}$ most common lethal gynecological malignancy with a 5 -year survival rate of about $47 \%$ and a localized stage diagnosis of $15 \%$, leading to about 125,000 global deaths each year. Therefore, it is urgent to explore novel and effective strategies for radical cure.

Methods: Short hairpin RNA targeting the Mucin16 (MUC16) gene was used to establish MUC16 knockdown in ovarian cancer cells. RT-PCR was performed to quantify the expression of MUC16 mRNA, and western blotting was performed to detect the expression of MUC16 and epithelial-mesenchymal transition-related proteins. Cell counting kit 8 (CCK8) wound healing and transwell assays were performed to assess cell proliferation and cell invasion. Flow cytometry was used to detect CD80-, CD83-, and CD86expressing dendritic cells (DCs) and cytotoxic T lymphocytes (CTLs) activated by MUC16-pulsed DCs.

Results: In this study, we identified MUC16 as a novel target antigen for immunotherapy against ovarian cancer, which was significantly up regulated in ovarian cancer cells and high-grade ovarian serous adenocarcinoma tissues. MUC16 knockdown in Ovcar3 cells using short hairpin RNA targeting the MUC16 gene suppressed the proliferation of migration, invasion, epithelial-mesenchymal transition (EMT), and PI3K/Akt signaling pathway in Ovcar3 cells markedly. MUC16 significantly up-regulated CD80, CD83, and CD86 (mature makers) expression in DCs and T-cell transformation into CD ${ }^{+}$T-cells detected by Flow cytometry.
\end{abstract}

Conclusions: For malignant ovarian cancer, MUC16 overexpression promoted cell proliferation, migration, and invasion via the PI3K/AKT signaling pathway. MUC16 pulsing mediated DC maturation and activated CTL response in vitro. Our study offers promising DC-based immunotherapy of considerable clinical value for patients with ovarian cancer.

Keywords: Ovarian cancer; Mucin16 (MUC16); dendritic cells (DCs) vaccine; cytotoxic T lymphocyte (CTL)

Submitted Aug 26, 2020. Accepted for publication Oct 30, 2020.

doi: 10.21037/atm-20-6388

View this article at: http://dx.doi.org/10.21037/atm-20-6388

\section{Introduction}

Cancer is still a significant health concern worldwide. Ovarian cancer is the fifth leading cause of death because of malignant diseases in women, leading to a predictable
125,000 global deaths each year, associated with the high mortality and poor prognosis among gynecological malignant tumors (1). Ovarian cancer shows high recurrence and mortality rates despite chemotherapy; therefore, a breakthrough in the treatment of this aggressive disease is

^ ORCID: 0000-0001-5141-4690. 
paramount (2).

With the first US FDA-approved dendritic cell (DC) vaccine for prostate cancer treatment, the DC-based vaccine has become a prospective new therapeutic option of cancer immunotherapy and has been presumed to be highly effective with minimal toxicity (3-7). As the natural professional antigen-presenting cells (APCs), DCs are incredibly efficient in phagocytosing, processing, and presenting antigens, and to activate the naïve resting $\mathrm{T}$-cells for anticancer immunity by transforming to the $\mathrm{CD}^{+}$or $\mathrm{CD}^{+} \mathrm{T}$ lymphocytes $(8,9)$. Clinically, after being injected into patients, the DC vaccines loaded with tumor antigens can migrate to the lymphoid tissues to elicit a robust antigen-specific T-cell response $(4,10)$.

Mucin16 (MUC16) is highly expressed in over $80 \%$ ovarian cancer tissues, while nearly undetected in normal ovarian tissues (11). CA125, encoded by MUC16, is the classic early stage circulating marker monitored in the clinical course of ovarian cancer patients $(12,13)$. MUC16 is a macromolecule transmembrane mucin comprising a single membrane-spanning domain, a cytoplasmic tail, and an extensive $\mathrm{N}$-terminal domain with a tandem repeat sequence (14), with CA125 antigen in the MUC16 tandem repeat (15). Therefore, MUC16 may serve as a promising novel target in anticancer therapies $(16,17)$. However, whether MUC16 can stimulate the cytotoxic T lymphocytes (CTLs) to kill the cancer cells delivered by the DC vaccine has not been reported in previous studies.

In this study, we investigated the role of MUC16 in ovarian cancer cells, and for the first time, the effect of MUC16 on DC-mediated immune responses was confirmed. Our results showed that MUC16 was up-regulated in the ovarian cancer cells and our locally collected ovarian cancer tissues to serve as an oncogenic gene and effective immunogenic antigen in ovarian cancer. MUC16 was delivered through the DC vaccine to stimulate the $\mathrm{CD}^{+} \mathrm{CTL}$ for the elimination of the tumor cells and offer promising and ideal immunotherapy for ovarian cancer.

We present the following article in accordance with the MDAR reporting checklist (available at http://dx.doi. org/10.21037/atm-20-6388).

\section{Methods}

\section{Antibodies and reagents}

Anti-MUC16 (1:1,000, Abcam), anti-PI3K (1:2,000, Abcam), anti- $\beta$-actin (1:5,000, Abcam) antibodies were used.

\section{Cell lines and cell culture}

Human ovarian cancer cell lines (Ovcar3, Skov3, and Caov3) were routinely supported and preserved in our laboratory. All cell lines were cultured in roswell park memorial institute (RPMI)-1640 medium holding 10\% fetal bovine serum (FBS) and $2 \mathrm{mM}$ L-glutamine at $37^{\circ} \mathrm{C}$ with $5 \% \mathrm{CO}_{2}$.

\section{MUC16 gene knockdown in Ovcar3 cells}

Three shRNA sequences targeting the MUC16 gene were designed: shRNA1 (5'-GCAGATACCAGTGCCATTAGACG AATCTAATGGCACTGGTATCTGC-3'), shRNA2 (5'-GGAGCAAACTGGGAAACTTCACGAATGA AGTTTCCCAGTTTGCTCC-3'), and shRNA3 (5'-GCAG CTGAACAACAGACAAGTCGAAACTTGTCTGTT GTTCAGCTGC-3'); shRNA-NC (5'-AAACGTGACAC GTTCGGAGAACGAATTCTCCGAACGTGT CACGTTT-3') was used as a control. The shRNA (5 $\mu \mathrm{g})$ and lentivirus expression plasmids were co-transfected into human embryonic kidney (HEK) 293T-cells to construct a MUC16 gene silencing lentivirus vector. Ovcar3 cells were then infected with the MUC16 lentivirus for 72 hours. Fluorescence microscopy detected the infection. Puromycin-selected cells were collected and used for later experiments. Knockdown efficiency was analyzed by immunoblotting with antibodies against MUC16.

\section{Western blotting}

Cells or ground tissues were dissolved in radio immunoprecipitation assay (RIPA) lysis buffer containing $1 \mathrm{mM}$ protease inhibitor cocktail and $1 \mathrm{mM}$ phenylmethanesulfonyl fluoride (PMSF). Cytoplasmic proteins were extracted using the cytoplasmic protein extraction kit (Beyotime Institute of Biotechnology, Haimen, China) according to the manufacturer's instructions. Proteins (10 $\mu \mathrm{g} /$ lane) were separated by $8 \%$ SDS-PAGE and transferred onto polyvinylidene fluoride (PVDF) membranes via electroblotting. The membranes were blocked with $5 \%$ bovine serum albumin in phosphate buffer saline (PBS) for 1 hour and then incubated with antibodies against $M U C 16(1: 1,000)$, phosphoinositide 3-kinase (PI3K, 1:2,000), or $\beta$-actin $(1: 5,000)$ overnight at $4{ }^{\circ} \mathrm{C}$. After washing in tris buffered saline tween (TBST, $50 \mathrm{mmol} / \mathrm{L}$ Tris, $145 \mathrm{mmol} / \mathrm{L} \mathrm{NaCl}$, and $0.05 \%$ Tween-20) three times for 10 minutes each, the membranes were 
incubated with secondary antibodies (1:5,000, Abcam) for 1 hour at room temperature. All experiments were repeated at least three times.

\section{$R N A$ extraction, reverse transcription (RT) and real-time PCR (RT-qPCR)}

Total RNA was isolated from cells or tissues using the TRIzol reagent (15596026; Thermo Fisher Scientific), and RT-qPCR was performed to synthesize cDNA with Prime Script RT reagent kit (Takara Bio Inc., Dalian, China) according to the manufacturer's instructions. Quantitative real-time PCR was performed using the SYBR Premix Ex Taq TM kit (Takara Bio Inc.) on a real-time PCR system. MUC16 expression was normalized to glyceraldehydephosphate dehydrogenase (GAPDH) expression and calculated using the $2^{-\Delta \Delta \mathrm{Ct}}$ method. The primer sequences used to amplify the target fragments were as follows: MUC16 forward, CCAGTCCTACATCTTCGGTTGT; MUC16 reverse, AGGGTAGTTCCTAGAGGGAGTT; GAPDH forward, GGAGCGAGATCCCTCCAAAAT; and GAPDH reverse, GGCTGTTGTCATACTT CTCATGG.

\section{Immunobistochemistry}

Paraffin-embedded tissue was cut to $4 \mu \mathrm{m}$ thick and blocked with endogenous peroxidase. Sections were then incubated overnight at $4{ }^{\circ} \mathrm{C}$ with an anti-MUC16 antibody at 1:50 dilution. Subsequently, the sections were washed three times with a biotin-labeled goat antibody. Sections were then stained with peroxidase-conjugated streptavidin with 3,3'-diaminobenzidine tetrahydrochloride as the chromogen. Finally, the sections were dyed with hematoxylin. The light microscopy captured each slide.

\section{Immunofluorescence microscopy}

Immunofluorescence microscopy was performed. Cells were seeded in 12 plants at $1 \times 10^{6}$ cells/well and incubated at $37^{\circ} \mathrm{C}$ overnight. Cells were fixed by $4 \%$ paraformaldehyde and permeabilized with methanol and then blocked by $3 \%$ BSA, and then incubated with anti-MUC16 antibody followed by incubation with CF568-conjugated goat-antirabbit IgG. Nuclei were revealed by diisopropanolamine (DIPA) staining ( $5 \mu \mathrm{g} / \mathrm{mL}$ in PBS) in the dark for 10 minutes at room temperature, and the cells were imaged using a confocal microscope.

\section{Cell proliferation, wound healing, and invasion assays}

Ovcar 3 cells were seeded in a 96-well plate $\left(2 \times 10^{3}\right.$ cells/well $)$ and observed at 4, 24, 48, and 72 hours. Cell counting kit 8 (CCK8) solution $(10 \mu \mathrm{L}$; Dojindo, Japan) was added to each well, and cells were incubated for 30 minutes at $37{ }^{\circ} \mathrm{C}$. Absorbance was quantified using a microplate spectrophotometer (Bio-Rad, USA) at $450 \mathrm{~nm}$.

For the wound healing assay, $3 \times 10^{6}$ cells were cultured to form a tight monolayer in a six-well plate for 24 hours. The cells were scraped using a sterile $200-\mu \mathrm{L}$ pipette tip to generate an artificial wound. The resulting gap (wound) between the cells was photographed using a microscope at 0 , $4,8,18,24$, and 48 hours. Migration capacity was evaluated by measuring the change in wound width. ImageJ was used to analyze the mean gap distance of the marginal cells in the scratched area (gate).

Invasion and migration assays were performed in trans-well chambers (8- $\mu \mathrm{m}$ pore size; Costar, Cambridge, MA, USA). For invasion assays, cells were seeded into trans-well chambers pre-coated with $40 \mu \mathrm{g} / \mathrm{mL}$ Matrigel (BD Biosciences, San Jose, CA, USA). For migration assays, cells were seeded into trans-wells without Matrigel coating. The upper chambers held RPMI-1640 medium supplemented with $0.1 \%$ FBS, and the lower chamber held RPMI-1640 medium supplemented with $20 \%$ FBS. After $48 \mathrm{~h}$ at $37^{\circ} \mathrm{C}$, the cells were fixed in paraformaldehyde (4\%) and stained with crystal violet. Noninvaded or non-migrated cells on the surface of the upper chambers were carefully removed with cotton swabs. The number of stained cells on the trans-well insert was observed and photographed under a light microscope. ImageJ was used to calculate the number of stained cells.

\section{Purification and cultivation of DCs and T-cells}

Monocytes were isolated from umbilical cord blood by centrifugation using ficoll (TBD, Tianjin, China). Umbilical cord blood was retrieved from full-term healthy pregnancies during cesarean sections. Research approval had been previously retrieved from the ethics committee of Beijing Chao-Yang Hospital (2020-science-154), and informed consent was retrieved from all the patients involved. The clinical study was conducted following the provisions of the Declaration of Helsinki (as revised in 2013). Red blood cells were removed using red blood cells lysis buffer (Solarbio, Beijing, China). Monocytes were washed with PBS (Life Technologies) and separated using a magnetic activated cell sorting (MACS) column with anti-CD14-conjugated 
microbeads (Miltenyi Biotec, Auburn, CA) according to the manufacturer's protocol. CD14 $4^{+}$monocytes were cultured in Iscove's modified Dulbecco's medium (Gibco, Gaithersburg, MD, USA) supplemented with 10\% FBS (HyClone, Logan, UT, USA), $100 \mathrm{U} / \mathrm{mL}$ recombinant human granulocyte colony-stimulating factor (rhGM-CSF), and $50 \mathrm{U} / \mathrm{mL}$ rhIL-4. CD14- mononuclear cells were cultured in the same medium supplemented with rhIL-2 $(20 \mathrm{ng} / \mathrm{mL})$ to induce the immature DCs.

\section{Sensitization of DCs}

Immature DCs were gathered on the fifth day and pulsed separately with the ovarian cancer antigen of recombinant human MUC16 protein (Fc Chimera Active) or PBS (control) for 48 hours. On the seventh day, DCs were harvested, washed, and stained with FITC-CD83 or PECD80 monoclonal antibodies, HLA-DR, CD11c, and corresponding isotype controls (BD Biosciences). Flow cytometry analyzed DCS (FACS Canton II, BD Biosciences).

\section{Generation and induction of CTLs}

DCs activated with MUC16 or PBS were harvested and separately co-cultured with T-cells in six-well plates at different ratios (DCs: T-cells, 1:5, 1:10, or 1:20) for six days. On days 1,3 , and 5 of incubation, T-cells were collected and labeled with human CD4-PE and CD8-FITC antibodies. The proportions of $\mathrm{CD}^{+} \mathrm{T}$ and $\mathrm{CD} 8^{+} \mathrm{T}$ lymphocytes were analyzed using flow cytometry and FlowJo.

\section{Cytotoxicity assay of CTLs}

CTLs (as the effector cell) and Ovcar3 or MUC16-KD Ovcar3 cells (as the target cell) co-cultured at different ratios (effector: target, 2.5:1, 5:1, 10:1, and 20:1) were seeded in a 96-well plate for 24 hours, with the effector cells or target cells alone as the control group. Cytotoxicity was examined using the CCK 8 reagent for 2 hours and analyzed as absorbance at $450 \mathrm{~nm}$. Cytotoxicity was calculated using the following formula: [(OD experimental group - OD effector cells alone group)/OD target cells alone group] $\times 100 \%$.

\section{Statistical analysis}

Experiments were performed three times independently. All the data in these experiments are available for providing to others for non-commercial use after the agreement of all authors. All data are presented as mean and standard deviation. Appropriately per experiment, results were analyzed using the nonparametric Mann-Whitney U test or analysis of variance with normal distributions to determine the significance of differences between groups. Values of $\mathrm{P}<0.05$ were considered statistically significant. All statistical analyses were performed using SPSS 22.0 (IBM Corporation, Armonk, NY, USA).

\section{Results}

\section{MUC16 expression in human ovarian cancer cell lines and local collected tissues}

We detected MUC16 expression levels in human ovarian cancer cell lines and local collected tissues. The mRNA and protein expression levels of MUC16 were highly upregulated in high-grade ovarian serous adenocarcinoma tissues, while were insignificant in control ovarian and the benign ovarian tissues. Image $\mathrm{J}$ software analyzed the gray ratio (Figure $1 A, B, C$ ). The immunohistochemical staining on the five pairs of human ovarian cancer tissues was performed. Our results showed that MUC16 protein expression in normal and benign ovarian tissues is lower than in OC tissues (Figure 1D).

Three human ovarian cancer cell lines (Ovcar3, Skov3, and Caov3) were then used to explore the biological function of MUC16 in ovarian cancer cellular development. MUC16 expression in Ovcar3 cells was higher than in Skov3 or Caov3 cells for both proteins (Figure 2A,B) and the mRNA levels (Figure 2C). Figure 2D shows the Immunofluorescence staining of MUC16 in ovarian cancer cell lines (Ovcar3, Skov3, and Caov3).

\section{MUC16 knockdown in an ovarian cancer cell line}

Ovcar3, an ovarian cancer cell line with high MUC16 expression, was selected to show a stable MUC16 knockdown cell line. Three specific short hairpin RNAs (shRNA01, shRNA02, and shRNA03) targeting the MUC16 gene were designed and synthesized to silence MUC16 expression in Ovcar3 cells. A scrambled shRNA served as negative control (NC). In Ovcar3 cells, shRNA02 significantly downregulated MUC16 protein expression $(\mathrm{P}<0.01)$; however, there was no significant difference in MUC16 protein expression between NC and Ovcar3 cells $(\mathrm{P}>0.05)$ (Figure 3A,B); therefore, shRNA02 was selected to establish a stable MUC16 knockdown ovarian cancer cell 
A

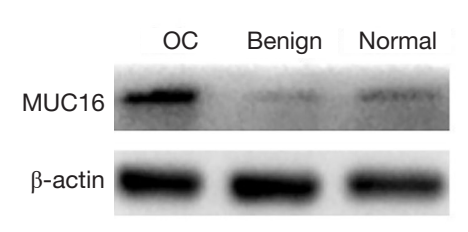

D
B

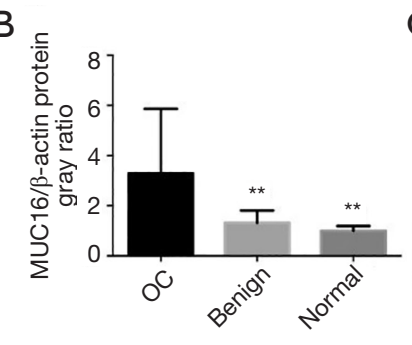

C

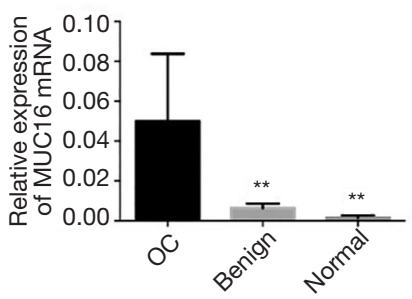

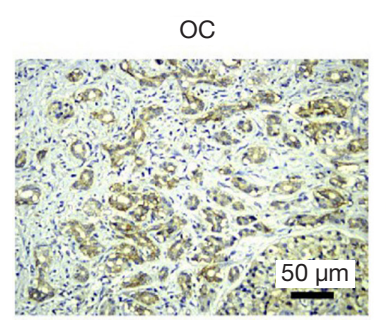
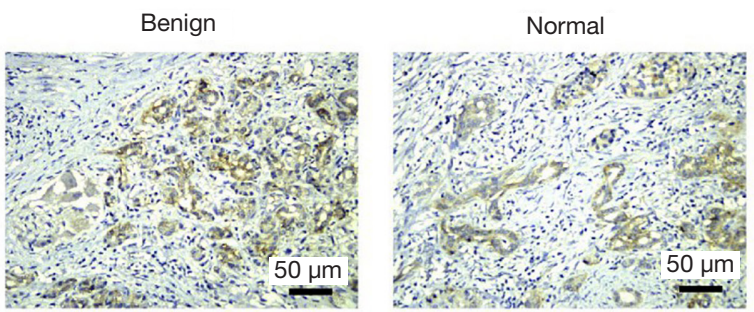

Figure 1 The expression levels of MUC16 protein and mRNA in human ovarian cancer tissues. $(\mathrm{A}, \mathrm{B})$ Western blot showing the relative MUC16 expression levels in ovarian cancer, benign ovarian, and normal ovarian tissues (OC, ovarian cancer; Benign, benign ovarian; Normal, normal ovarian). (C) RT-qPCR shows the relative mRNA MUC16 expression levels in ovarian cancer, benign ovarian, and normal ovarian tissues. (D) Immunohistochemical staining of MUC16 in ovarian cancer, benign ovarian, and normal ovarian tissues. The bar chart is a statistical analysis from the results of three independent experiments. Data are expressed as mean \pm SEM. ${ }^{* *}, \mathrm{P}<0.05$.

line (MUC16-KD).

\section{Effects of MUC16 knockdown on invasion, proliferation, and migration of ovarian cancer cells in vitro}

To identify the function of the MUC16 gene in Ovcar3 cells, we investigated the ability of NC Ovcar3 and MUC16-KD cells to migrate and invade using a trans-well assay. The invasive ability of MUC16-KD Ovcar3 cells was markedly suppressed compared with $\mathrm{NC}$ Ovcar3 cells $(\mathrm{P}<0.01)$ (Figure $4 A, B)$. MUC16 knockdown inhibited cell proliferation, as shown by the cell growth curves of CCK 8 assay $(\mathrm{P}<0.01$; Figure $4 C$ ). These results altogether showed MUC16 silencing inhibited cell migration $(\mathrm{P}<0.01$; Figure $4 D, E)$.

\section{Effects of MUC16 knockdown on epithelial-mesenchymal transition (EMT) and the PI3K/Akt signaling pathway in Ovcar3 cells}

EMT is a process through which epithelial cells acquire a mesenchymal phenotype. During EMT, cells gradually lose the epithelial characteristics of cell-to-cell adhesion and apicobasal polarity to reorganize the cytoskeleton into a spindle-like cell shape, thereby gaining increased motility and invasiveness. Hence, EMT is considered a critical process in tumor invasion. Common molecular markers for EMT include the losing expression of epithelial markers (e.g., E-cadherin) and gaining expression of mesenchymal markers (e.g., $\mathrm{N}$-cadherin and vimentin). We, therefore, used MUC16-KD and NC Ovcar3 cells to investigate the mechanism of MUC16-mediated regulation of cell invasion. The expression of EMT markers (E-cadherin, $\mathrm{N}$-cadherin, and vimentin) was detected by western blotting. E-cadherin expression was increased, but $\mathrm{N}$-cadherin and vimentin expression was decreased in MUC16-KD cells compared with those in NC Ovcar3 cells (Figure 5A,B).

The PI3K/Akt signaling pathway is vital for cancer cell invasion and metastasis through EMT regulation. Thus, we further evaluated the function of MUC16 in PI3K/ Akt signaling pathway activation in Ovcar3 cells. MUC16 downregulation significantly reduced $\mathrm{p}-\mathrm{PI} 3 \mathrm{~K}$ and $\mathrm{p}-\mathrm{Akt}$ expression (Figure 5C,D). These results suggested that MUC16 downregulation inhibited EMT and the PI3K/Akt signaling pathway in human ovarian cancer cells.

\section{Effects of MUC16 stimulation on DC maturation}

CD $14^{+}$monocytes isolated from umbilical cord blood were cultured with $100 \mathrm{U} / \mathrm{mL}$ recombinant human GMCSF (rhGM-CSF) and $50 \mathrm{U} / \mathrm{mL} \mathrm{IL-4} \mathrm{(rhIL-4)} \mathrm{to} \mathrm{produce}$ 
A

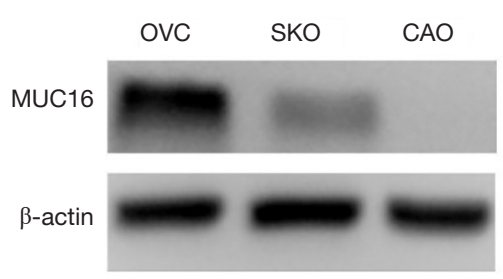

D
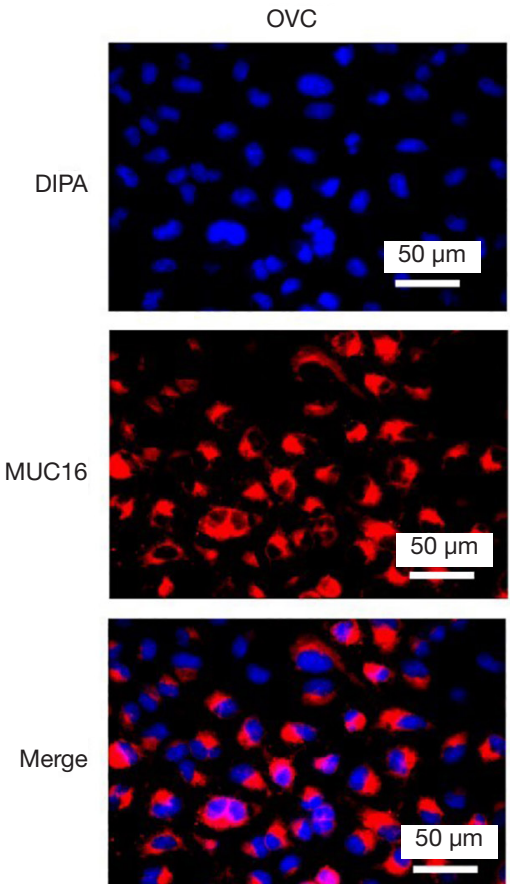

B

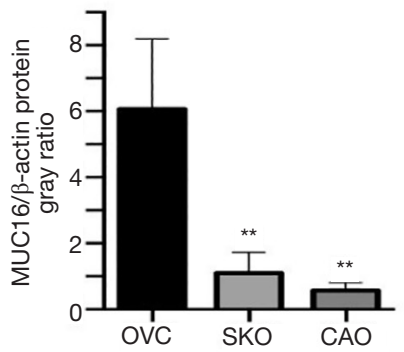

SKO
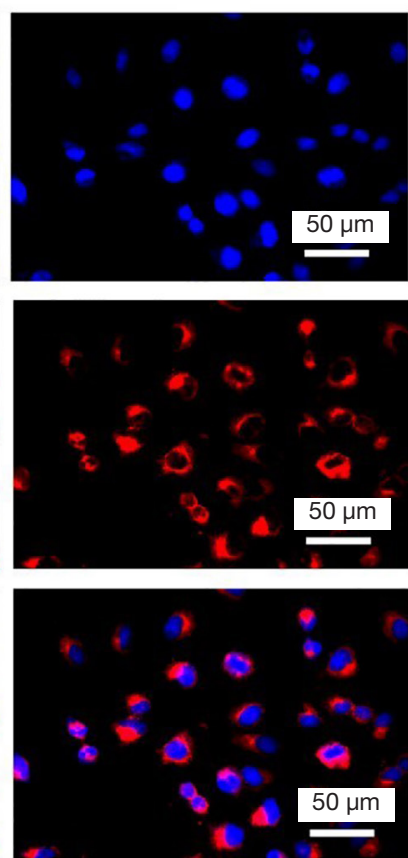

C

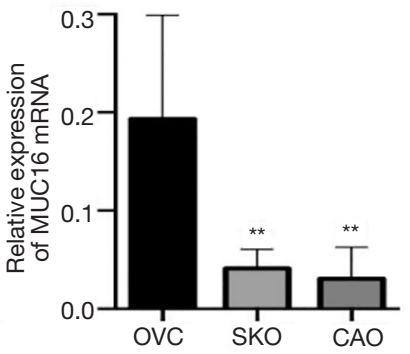

$\mathrm{CAO}$
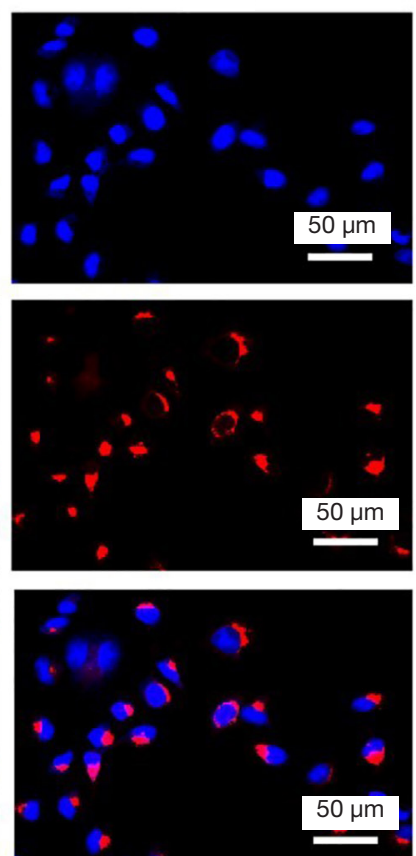

Figure 2 The expression of MUC16 protein and mRNA in human ovarian cancer cell lines. (A,B) Western blot showing the MUC16 expression levels in ovarian cancer cell lines (OVC: Ovcar3; SKO: Skov3; CAO: Caov3). (C) RT-qPCR shows the relative MUC16 mRNA expression levels in ovarian cancer cell lines (Ovcar3, Skov3, and Caov3). (D) Immunofluorescence staining of MUC16 in ovarian cancer cell lines (Ovcar3, Skov3, or Caov3). The bar chart is a statistical analysis from the results of three independent experiments. Data are expressed as mean \pm SEM. ${ }^{* *}, \mathrm{P}<0.05$.

monocyte-derived DCs. On the fifth day, DCs were stimulated with recombinant MUC16 or PBS for 48 hours. Recombinant MUC16 stimulation significantly up-regulated CD80, CD83, and CD86 (mature makers) expression in DCs compared with that in the controls (Figure 6), indicating an efficient cancer antigen of the MUC16 in ovarian cancer.

\section{The ability of MUC16-pulsed DCs to promote T-cell transformation into $\mathrm{CD} 8^{+} \mathrm{T}$-cells}

Mature DCs can sensitize and activate T lymphocytes (5).
Thus, we evaluated the activation ability of MUC16presenting DCs, as assessed by the number of $\mathrm{CD}^{+}{ }^{+} \mathrm{T}$-cells. PBS-loaded DCs or MUC16-pulsed DCs were co-cultured with $\mathrm{T}$ lymphocytes at a ratio of 5:1, and the morphology of activated T-cells was observed using an Olympus microscope (Figure 7A). The percentage of $\mathrm{CD}^{+} \mathrm{T}$-cells was measured via flow cytometry (Figure 7B). MUC16pulsed DCs induced more CD8 ${ }^{+}$T-cells than PBS-loaded DCs (Figure 7C). According to the results, MUC16-pulsed DCs can induce a strong activation function compared to the controls. 


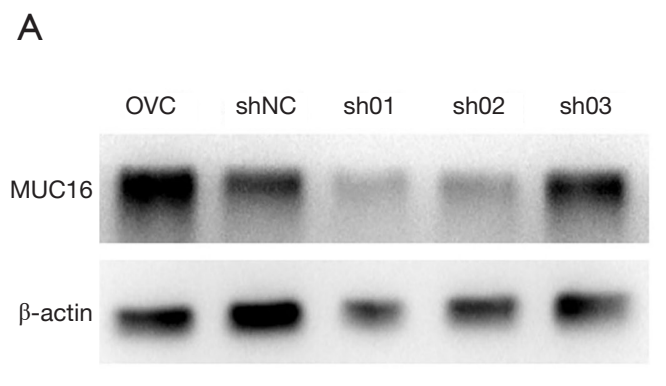

B

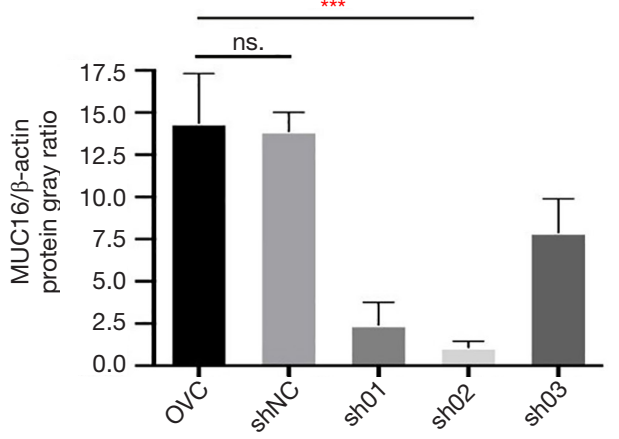

Figure 3 Establishment of stable MUC16 knockdown ovarian cancer cells. (A,B) Western blot showed the MUC16 expression levels in three short hairpin RNAs, negative control, and ovcar3. The bar chart is a statistical analysis from the results of three independent experiments. Data are expressed as mean \pm SEM. ${ }^{* * *}, \mathrm{P}<0.01$.

\section{Effects of MUC16-pulsed DCs on the cytotoxicity of T-cells against ovarian cancer cells}

Ovcar3 cells were used as targets and T lymphocytes as effectors to investigate whether MUC16-pulsed DCs induced a specific CTL response in vitro. A dose-dependent cytotoxic effect against Ovcar3 cells was examined at various ratios (T-cells: Ovcar3 cells $=2.5: 1,5: 1,10: 1$, or 20:1). The cytotoxicity of CTLs induced by MUC16-pulsed DCs was significantly higher than CTLs induced by PBS-loaded DCs at the ratio of 20:1 (Figure 8). These data showed that MUC16-pulsed DCs induced potent and specific cytotoxicity against ovarian cancer cells in vitro.

\section{Discussion}

MUC16 is a classic biomarker for ovarian cancer owing to its aberrant expression in this malignancy. From its transmembrane structure with different domains coordinately mediating various signaling pathways, MUC16 may bear more responsibilities rather than only being a biomarker (14). MUC16 has been reported to promote cancer cell progression by stimulating cell adhesion, growth, and metastasis, and evading attacks from natural killer cells (18-21). In this study, we further confirmed the function of MUC16 to promote ovarian cancer cell proliferation, migration, and invasion, which was triggered by the activation of the PI3K/AKT signaling pathway.

The transformation of the immature antigen-capturing DCs into the mature antigen-presenting DCs is the crucial process in preparing DC-loaded antigen vaccination, endowed with the ability to interact with T-cells and initiate specific immune responses $(3,4,10)$. Several coordinated events accompany DC maturation, including the movement of $\mathrm{MHC} /$ peptide complexes to the cell membrane, upregulation of the classic markers for DC maturation, including the costimulatory molecules (CD40, CD83, CD80, and CD86) (9). In current work, we found that the recombinant MUC16 stimulation significantly up-regulated the mature makers of DCs, including the CD80, CD83, and CD86, which indicated the MUC16 could be an efficient antigen of ovarian cancer and designated it as an ideal target for immunotherapy against ovarian cancer. Currently, the molecular mechanisms underlying the effect of MUC16 on the maturation of DCs and activation of CTL activity in the progression of cancers remain unknown. MUC16 is involved in a variety of signaling pathways affecting the cellular proliferation, immigration, as well as invasion. For example, MUC16 regulates the activity of cervical cancer cells through the JAK2/STAT3 pathway (22). More importantly, Xin et al. (23) found that MUC16 played a role in promoting the $\mathrm{p} 120$-catenin to translocate to the cytoplasm, thereby activating the RhoA/Cdc42 to enhance the ability of migration and proliferation of ovarian cancer cells. Here, we found that the expression of PI3K/Akt were decreased in the MUC16-knockdown group, suggesting a role of PI3K/Akt pathway involved in the effect of MUC16 on ovarian cancer cells. However, more studies such as inhibiting the PI3K/Akt pathway should be conducted to further confirm the involvement of PI3K/Akt in these processes in the future. Though the MUC16 binds siglecs and masks Toll-like receptors (TLRs) on DCs, promoting an immature DC phenotype that, in turn, decreases $\mathrm{T}$ cell effector functions have been reported (24). However, the detection of circulating MUC16-specific antibodies 
A

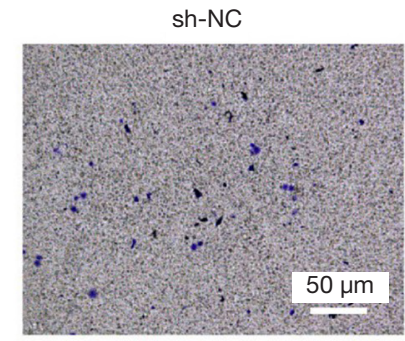

B

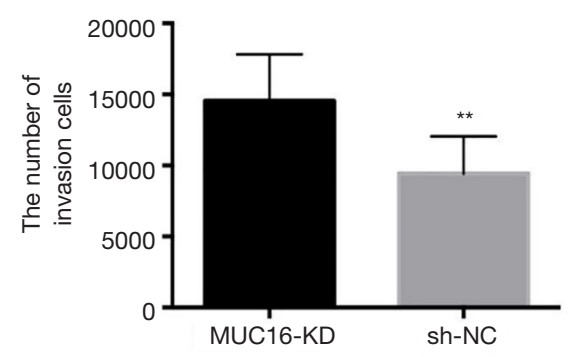

D
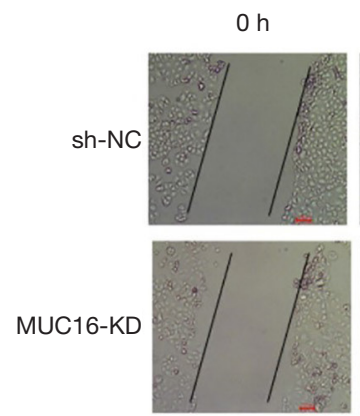

$6 \mathrm{~h}$

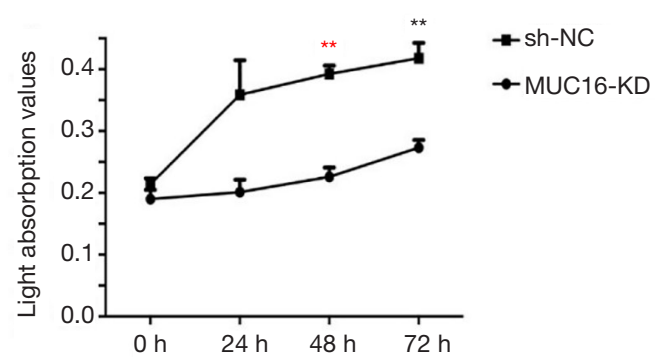

E

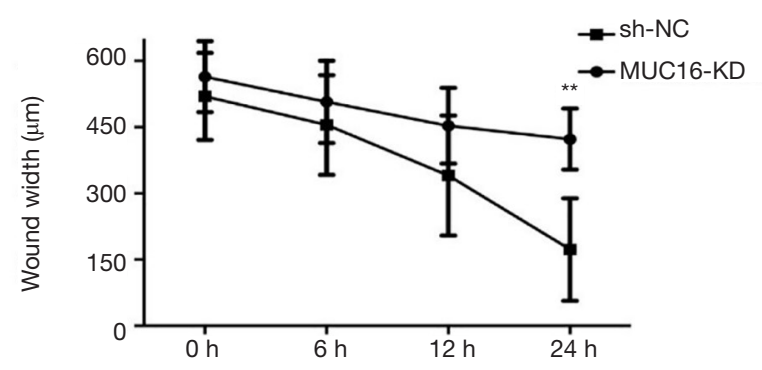

Figure 4 Down-regulation of MUC16 inhibits invasion, proliferation, and migration of ovarian cancer cells in vitro. (A,B) The Transwell showed that MUC16 silencing in ovcar3 cells suppressed the invasive ability of ovcar3 cells. Significantly decreased invasion was observed in MUC16-KD ovcar3 cells than in NC cells $(9,424 \pm 829.6$ vs. $14,554 \pm 1,036, \mathrm{P}<0.01)$. (C) According to the cell growth curves of CCK-8 cell proliferation assay, the MUC16 deficiency inhibited the proliferation of ovcar3 cells $(\mathrm{P}<0.01)$. (D,E) Wound-healing cell migration assay in ovcar 3 cells following MUC16-KD. MUC16-KD inhibited cell migration and wounding-healing repairing $(\mathrm{P}<0.01)$. Scale bar $=50 \mu \mathrm{m}$. ${ }^{* *}$, $\mathrm{P}<0.05$. 
A

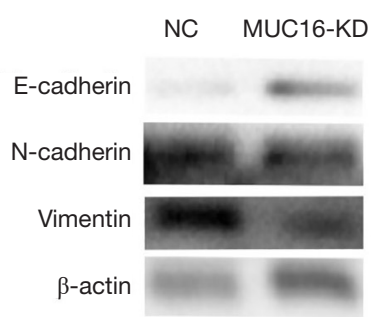

B

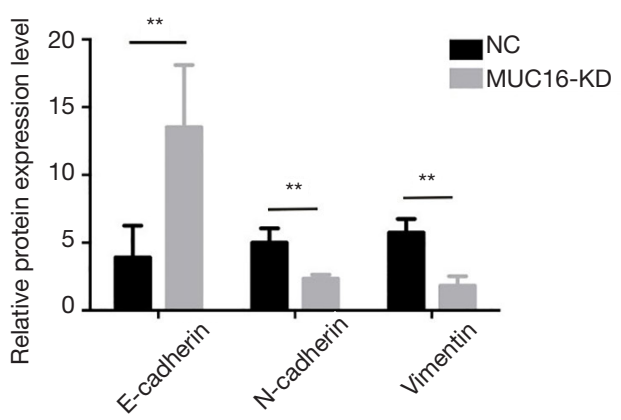

C

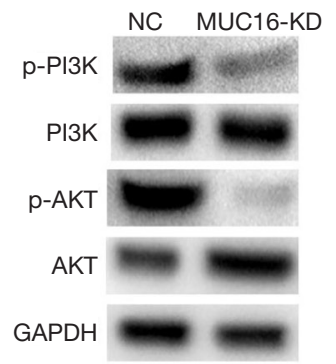

D

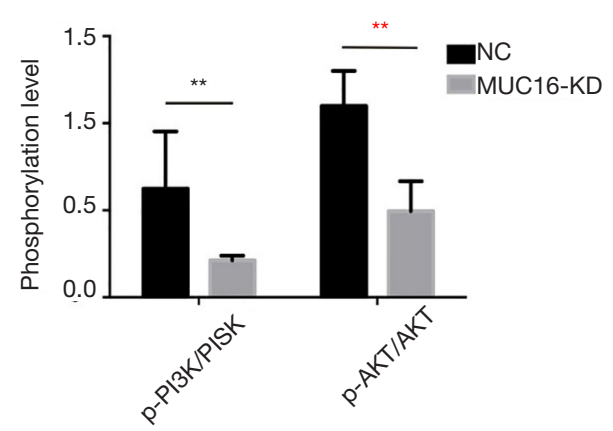

Figure 5 Down-regulation of MUC16 inhibits the epithelial-mesenchymal transition (EMT) process, and the PI3K/Akt signaling pathway takes part in the inhibitory effect in ovarian cancer cells. (A,B) The protein levels of EMT-related markers by western blot analysis in MUC16-KD ovcar3 cells compared with corresponding NC cells $(\mathrm{P}<0.05)$. (C,D) Down-regulation of MUC16 decreased the protein expression of $\mathrm{p}-\mathrm{PI} 3 \mathrm{~K}$ and $\mathrm{p}-\mathrm{Akt}$ in ovcar3 cells $(\mathrm{P}<0.05)$. ${ }^{* *}, \mathrm{P}<0.05$.

A
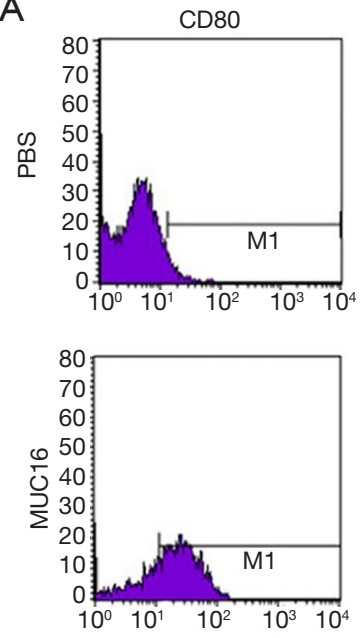
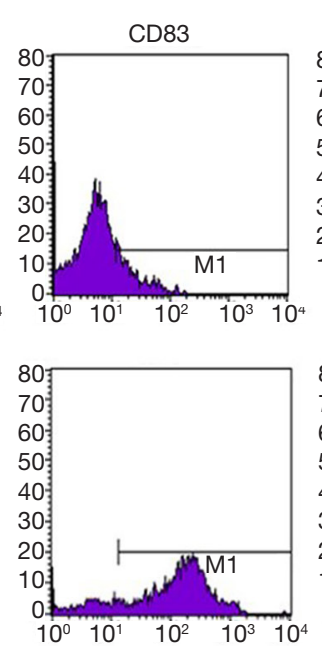
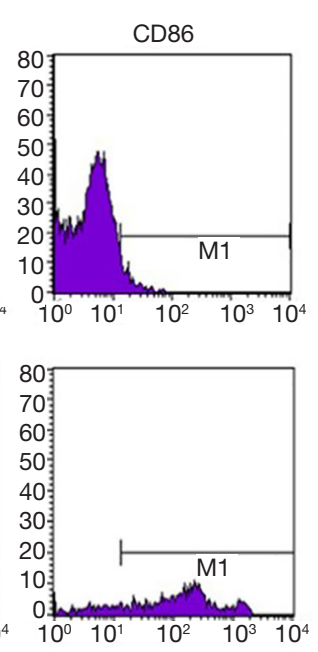

B

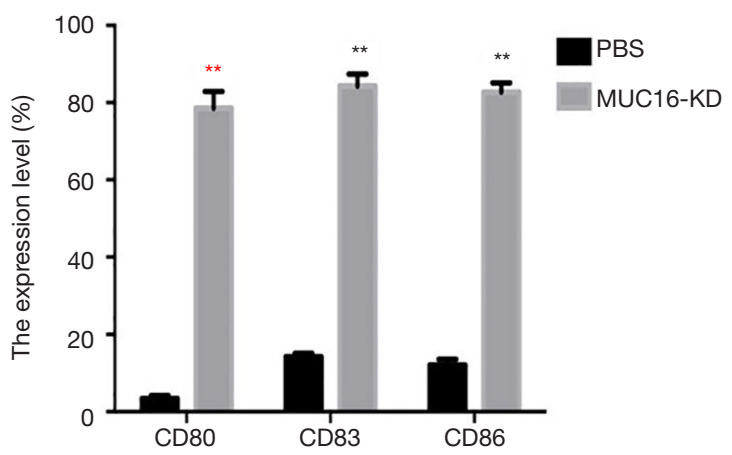

Figure $6 \mathrm{CD}_{14} 4^{+}$monocytes stimulated by MUC16 acquired dendritic cells. (A,B) $\mathrm{CD} 14^{+}$monocytes cultured for 5 days with rhGM-CSF and rhIL-4, and another 48h with the cancer antigen of recombinant MUC16, which significantly up-regulated the expression of CD80, CD83, and CD86 (the mature DCs) compared with phosphate buffer saline (PBS) $(\mathrm{P}<0.05)$. ${ }^{* *}, \mathrm{P}<0.05$. 
A

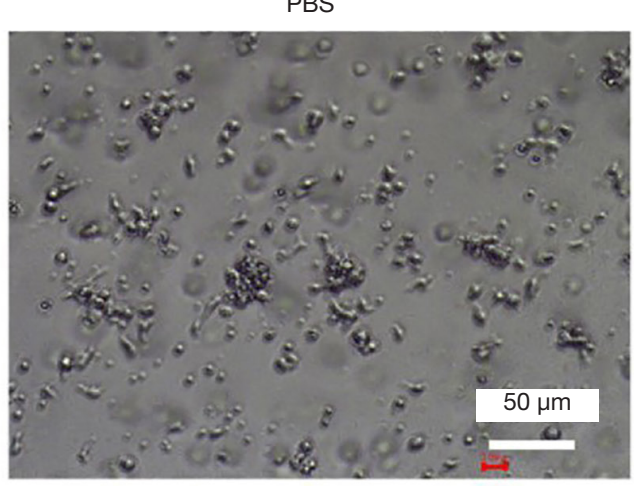

MUC16

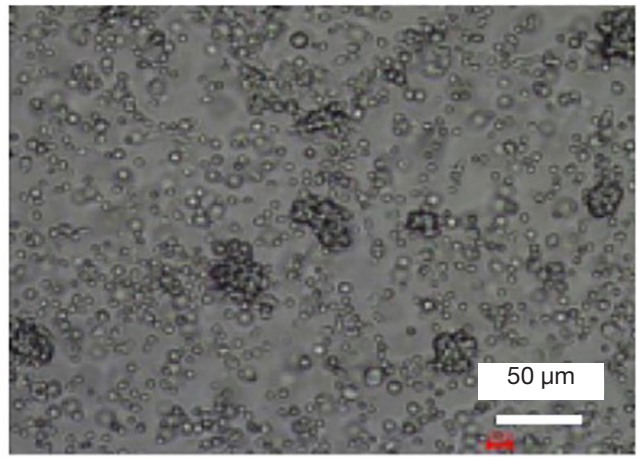

B

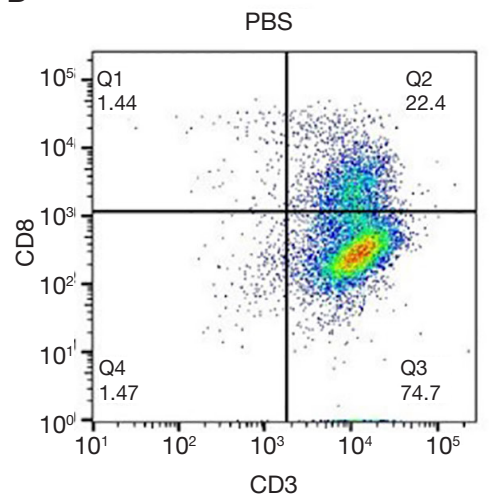

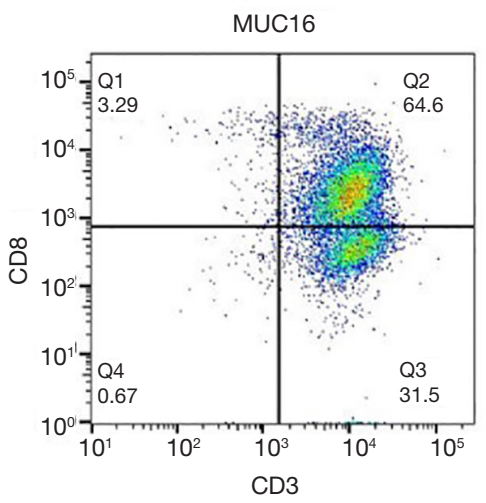

C

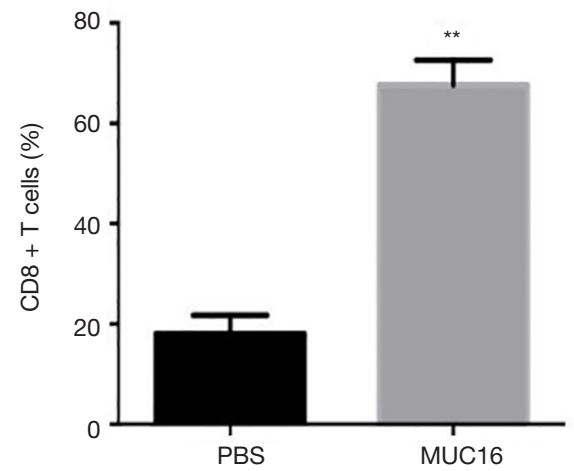

Figure 7 Mature dendritic cells (DCs) activated T lymphocytes and induced CD8+ T cells. (A) Mature DCs activated T lymphocytes at the ratio of 1:5 (×10). (B) MUC16-pulsed DCs induced more CD8+ T cells than phosphate buffer saline (PBS)-loaded DCs (P<0.05). (C) The statistical analysis is from (B) results of three independent experiments. Data are expressed as mean $\pm \mathrm{SEM} .{ }^{* *}, \mathrm{P}<0.05$.

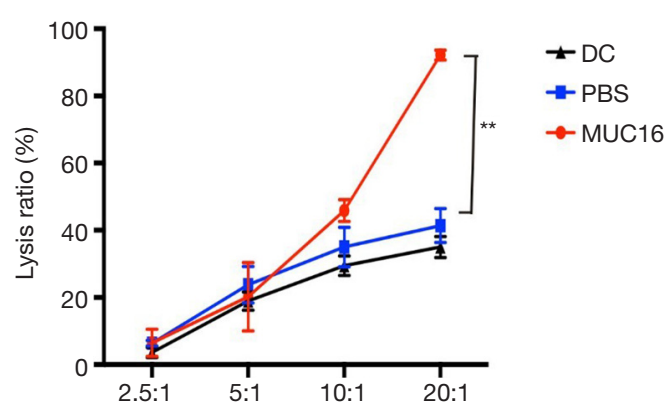

Figure 8 MUC16 pulsed dendritic cells (DCs) to activate T cells and trigger cytotoxic lymphocytic cell responses on ovarian cancer cells in vitro. When T cells and OVC cells at ratio 20:1, the cytotoxicity of cytotoxic T lymphocytes (CTLs) against target cells induced by MUC16-pulsed DCs was more substantial than that induced by phosphate buffer saline (PBS)-pulsed DCs (**, $\mathrm{P}<0.05)$.

in cancer patients indicates their immunogenic potential in stimulating antigen-specific humoral responses $(25,26)$.
Moreover, a recent study published in Nature identified MUC16 as a hotspot with a 4-fold higher neoantigen frequency in the long-term survivors of pancreatic cancer as versus the short-term survivors (27). Meanwhile, though the receptor-based endocytosis involving TLRs, Fc receptors, integrins, $C$-type lectins, and scavenger receptors, DCs internalize exogenous antigens for the processing and presentation (9). Notably, the C-type lectin receptors (CLRs) recognize carbohydrate structures containing antigens, which strongly indicates CLRs of DCs may positively recognize MUC16 for the phagocytose, the antigen processing, and presentation. Through this process, the extracellular MUC16 peptide (CA125 antigen) would primarily present by cross-presentation, a particular capacity of DCs for presenting exogenous antigenic peptides to activate $\mathrm{CD}^{+} \mathrm{T}$-cells via $\mathrm{MHC}$-I directly.

DCs in the peripheral blood may also affect the activity of ovarian cancer cells by regulating the immune responses. Previous study showed that number of peripheral DCs was 
increased by Fms-like tyrosine kinase 3 ligand (FLT3L), and thereby enhancing the in vivo immunity against the tumors (28). In ovarian cancer, FLT3L can increase the expression of CD40 in DCs and activates cytotoxic T lymphocytes (29). More and more scholars believed that DC-targeted immunotherapy can be a promising strategy for the treatment of ovarian cancer. The advantages favoring DC-based vaccines over other immunotherapies include the well-tolerated Nature and minimal toxicity, which inspire further exploration to optimize their effects, mainly by improving the antigen-loading methods and capacity of DC cell migration to lymphoid tissues, as well as optimizing tumor microenvironment. However, DC vaccines attacking tumor cells may be blocked by the immune-evasive tumor microenvironment, including insufficient tumor antigens, secretion of immunosuppressive cytokines, increase in the number of inhibitory ligands, and induction of Treg cells. This urges the investigation of DC vaccines combined with other strategies to overcome these barriers. Indeed, there have been several successful trials on the combination of DCs and CTLs with cytokine-induced killer therapy, as well as the DC vaccines with photodynamic therapy for enhanced antigen presentation (30,31).

In conclusion, the data in our study showed that MUC16 served as an oncogenic gene and an effective immunogenic antigen in ovarian cancer. MUC16 delivered through the DC vaccine could boost an endogenous antitumor response and stimulate the $\mathrm{CD} 8^{+}$CTLs to lysis the ovarian cancer cells, which may provide an effective therapeutic strategy against ovarian cancer in the clinical setting.

\section{Acknowledgments}

Funding: This work was supported by the Beijing Municipal Administration of Hospitals Clinical Medicine Development of Special Funding-Yangfan Project (no. ZYLX201713) and China National Natural Science Foundation (no. 81571455).

\section{Footnote}

Reporting Checklist: The authors have completed the MDAR reporting checklist. Available at http://dx.doi.org/10.21037/ atm-20-6388

Data Sharing Statement: Available at http://dx.doi. org/10.21037/atm-20-6388

Conflicts of Interest: All authors have completed the ICMJE uniform disclosure form (available at http://dx.doi. org/10.21037/atm-20-6388). The authors have no conflicts of interest to declare.

Ethical Statement: The authors are accountable for all aspects of the work in ensuring that questions related to the accuracy or integrity of any part of the work are appropriately investigated and resolved. The clinical study was approved by the Ethics Committee of Beijing ChaoYang Hospital (2020-science-154) and was conducted following the provisions of the Declaration of Helsinki (as revised in 2013). Written informed consent was retrieved from all participants before enrollment.

Open Access Statement: This is an Open Access article distributed in accordance with the Creative Commons Attribution-NonCommercial-NoDerivs 4.0 International License (CC BY-NC-ND 4.0), which permits the noncommercial replication and distribution of the article with the strict proviso that no changes or edits are made and the original work is properly cited (including links to both the formal publication through the relevant DOI and the license). See: https://creativecommons.org/licenses/by-nc-nd/4.0/.

\section{References}

1. Siegel RL, Miller KD, Jemal A. Cancer statistics, 2018. CA Cancer J Clin 2018;68:7-30.

2. Gorodnova TV, Sokolenko AP, Kuligina E, et al. Principles of clinical management of ovarian cancer. Chin Clin Oncol 2018;7:56.

3. Galati D, Zanotta S. Empowering dendritic cell cancer vaccination: the role of combinatorial strategies. Cytotherapy 2018;20:1309-23.

4. Gardner A, Ruffell B. Dendritic Cells and Cancer Immunity. Trends Immunol 2016;37:855-65.

5. Constantino J, Gomes C, Falcao A, et al. Dendritic cellbased immunotherapy: a basic review and recent advances. Immunol Res 2017;65:798-810.

6. Jung NC, Lee JH, Chung KH, et al. Dendritic CellBased Immunotherapy for Solid Tumors. Transl Oncol 2018;11:686-90.

7. Lu H, Dai X, Li X, et al. Gal-1 regulates dendritic cellsinduced Treg/Th17 balance though NF-kappaB/RelBIL-27 pathway. Ann Transl Med 2019;7:628.

8. Ballestrero A, Boy D, Moran E, et al. Immunotherapy with dendritic cells for cancer. Adv Drug Deliv Rev 2008;60:173-83. 
9. Banchereau J, Briere F, Caux C, et al. Immunobiology of dendritic cells. Annu Rev Immunol 2000;18:767-811.

10. Sabado RL, Bhardwaj N. Dendritic cell immunotherapy. Ann N Y Acad Sci 2013;1284:31-45.

11. Bast RC Jr, Klug TL, St John E, et al. A radioimmunoassay using a monoclonal antibody to monitor the course of epithelial ovarian cancer. N Engl J Med 1983;309:883-7.

12. Bast RC Jr, Feeney M, Lazarus H, et al. Reactivity of a monoclonal antibody with human ovarian carcinoma. J Clin Invest 1981;68:1331-7.

13. Yin BW, Lloyd KO. Molecular cloning of the CA125 ovarian cancer antigen: identification as a new mucin, MUC16. J Biol Chem 2001;276:27371-5.

14. Kufe DW. Mucins in cancer: function, prognosis and therapy. Nat Rev Cancer 2009;9:874-85.

15. Aithal A, Rauth S, Kshirsagar P, et al. MUC16 as a novel target for cancer therapy. Expert Opin Ther Targets 2018;22:675-86.

16. Das S, Batra SK. Understanding the Unique Attributes of MUC16 (CA125): Potential Implications in Targeted Therapy. Cancer Res 2015;75:4669-74.

17. Felder M, Kapur A, Gonzalez-Bosquet J, et al. MUC16 (CA125): tumor biomarker to cancer therapy, a work in progress. Mol Cancer 2014;13:129.

18. Belisle JA, Horibata S, Jennifer GA, et al. Identification of Siglec-9 as the receptor for MUC16 on human NK cells, B cells, and monocytes. Mol Cancer 2010;9:118.

19. Thériault C, Pinard M, Comamala M, et al. MUC16 (CA125) regulates epithelial ovarian cancer cell growth, tumorigenesis and metastasis. Gynecol Oncol 2011;121:434-43.

20. Chen X, Li X, Wang X, et al. MUC16 impacts tumor proliferation and migration through cytoplasmic translocation of P120-catenin in epithelial ovarian cancer cells: an original research. BMC Cancer 2019;19:171.

21. Haridas D, Ponnusamy MP, Chugh S, et al. MUC16: molecular analysis and its functional implications in benign and malignant conditions. FASEB J 2014;28:4183-99.

22. Hui S, Meng G, Lu W, et al. MUC16 facilitates cervical cancer progression via JAK2/STAT3 phosphorylation-

Cite this article as: Zhai Y, Lu Q, Lou T, Cao G, Wang S, Zhang Z. MUC16 affects the biological functions of ovarian cancer cells and induces an antitumor immune response by activating dendritic cells. Ann Transl Med 2020;8(22):1494. doi: 10.21037/atm-20-6388 mediated cyclooxygenase-2 expression. Genes Genomics 2020;42:127-33.

23. Xin C, Xiaoduan L, Xinjing $W$ et al., MUC16 impacts tumor proliferation and migration through cytoplasmic translocation of P120-catenin in epithelial ovarian cancer cells: an original research. BMC Cancer 2019;19:171.

24. Bhatia R, Gautam SK, Cannon A, et al. Cancer-associated mucins: role in immune modulation and metastasis.

Cancer Metastasis Rev 2019;38:223-36.

25. Marcos-Silva L, Ricardo S, Chen K, et al. A novel monoclonal antibody to a defined peptide epitope in MUC16. Glycobiology 2015;25:1172-82.

26. Blixt O, Bueti D, Burford B, et al. Autoantibodies to aberrantly glycosylated MUC1 in early stage breast cancer are associated with a better prognosis. Breast Cancer Res 2011;13:R25.

27. Balachandran VP, Luksza M, Zhao JN, et al. Identification of unique neoantigen qualities in long-term survivors of pancreatic cancer. Nature 2017;551:512-6.

28. Masten BJ, Olson GK, Kusewitt DF et al. Flt3 ligand preferentially increases the number of functionally active myeloid dendritic cells in the lungs of mice. J Immunol. 2004;172:4077-83.

29. Matsumura N, Mandai M, Hamanishi J, et al. Immunostimulatory effect of Fms-like tyrosine kinase 3 ligand on peripheral monocyte-derived dendritic cells and natural killer cells: utilization for ovarian cancer treatment. Oncol Rep 2008;19:505-15.

30. Zhang H, Wang P, Wang X, et al. Antitumor Effects of DC Vaccine With ALA-PDT-Induced Immunogenic Apoptotic Cells for Skin Squamous Cell Carcinoma in Mice. Technol Cancer Res Treat 2018;17:1533033818785275.

31. Wang Y, Xu Z, Zhou F, et al. The combination of dendritic cells-cytotoxic $\mathrm{T}$ lymphocytes/cytokine-induced killer (DC-CTL/CIK) therapy exerts immune and clinical responses in patients with malignant tumors. Exp Hematol Oncol 2015;4:32.

(English Language Editor: J. Chapnick) 